\title{
Intramolecular Diffusive Motion in Alkane Monolayers Studied by High-Resolution Quasielastic Neutron Scattering and Molecular Dynamics Simulations
}

\author{
F. Y. Hansen, ${ }^{1}$ L. Criswell, ${ }^{2}$ D. Fuhrmann, ${ }^{2, *}$ K.W. Herwig, ${ }^{3}$ A. Diama, ${ }^{2}$ R. M. Dimeo, ${ }^{4}$ D. A. Neumann, ${ }^{4}$ \\ U. G. Volkmann, ${ }^{5}$ and H. Taub ${ }^{2}$ \\ ${ }^{1}$ Department of Chemistry, Technical University of Denmark, IK 207 DTU, DK-2800 Lyngby, Denmark \\ ${ }^{2}$ Department of Physics and Astronomy and University of Missouri Research Reactor, University of Missouri-Columbia, \\ Columbia, Missouri 65211, USA \\ ${ }^{3}$ Spallation Neutron Source, Oak Ridge National Laboratory, Oak Ridge, Tennessee 37830, USA \\ ${ }^{4}$ NIST Center for Neutron Research, National Institute of Standards and Technology, Gaithersburg, Maryland 20899-8562,USA \\ ${ }^{5}$ Facultad de Física, Pontificia Universidad Católica de Chile, Santiago 22, Chile
}

(Received 6 June 2003; published 29 January 2004)

\begin{abstract}
Molecular dynamics simulations of a tetracosane $\left(n-\mathrm{C}_{24} \mathrm{H}_{50}\right)$ monolayer adsorbed on a graphite basalplane surface show that there are diffusive motions associated with the creation and annihilation of gauche defects occurring on a time scale of $\sim 0.1-4 \mathrm{~ns}$. We present evidence that these relatively slow motions are observable by high-energy-resolution quasielastic neutron scattering (QNS) thus demonstrating QNS as a technique, complementary to nuclear magnetic resonance, for studying conformational dynamics on a nanosecond time scale in molecular monolayers.
\end{abstract}

DOI: 10.1103/PhysRevLett.92.046103

PACS numbers: 68.35.Ja, 61.12.-q, 68.35.Fx, 87.15.He

Intermediate-length alkane molecules $\left(\mathrm{C}_{\mathrm{n}} \mathrm{H}_{2 \mathrm{n}+2} ; 15<\right.$ $\mathrm{n}<35$ ) adsorbed on solid substrates offer attractive model systems for studying a wide range of interfacial phenomena. Such alkanes are believed to be large enough to exhibit some features of more complex adsorbed polymers and yet small enough to be tractable in detailed molecular dynamics (MD) simulations. They have been used to address a variety of issues of current interest including polymer film growth [1], selective adsorption of polymers [2], microscopic mechanisms of friction and lubrication [3], and the gel-to-fluid transition in bilayer lipid membranes [4].

In the last example, we refer to MD simulations and neutron diffraction measurements [4] that suggest the melting transition in monolayers of tetracosane $\left(n-\mathrm{C}_{24} \mathrm{H}_{50}\right.$ or $\left.\mathrm{C} 24\right)$ and dotriacontane $\left(n-\mathrm{C}_{32} \mathrm{H}_{66}\right.$ or $\left.\mathrm{C} 32\right)$ adsorbed on a graphite basal-plane surface to be analogous to the gel-to-fluid transition in bilayer lipid membranes [5]. That is, MD simulations show evidence of monolayer melting in which intramolecular and translational order are lost simultaneously. Figure 1 presents top views of the C24 molecules in the MD simulation cell both above and below the transition temperature at $\sim 340 \mathrm{~K}$ calculated in our earlier work [4]. At $230 \mathrm{~K}$ (left panel), we see translational order manifested by the lamellar structure of the monolayer [6]. Since both the simulations and quasielastic neutron scattering (QNS) measurements (see below) indicate some translational diffusive motion within the lamellae at this temperature, we refer to this structure as the "smectic" phase. At $350 \mathrm{~K}$ (right panel), the individual molecules have transformed to a more globular shape; i.e., they undergo "chain melting," and the monolayer has lost its translational order characterized by the lamellar structure. As in the case of the gel-to-fluid transition in bilayer lipid membranes, the MD simulations demonstrate [4] that the simultaneous chain and lattice melting result from an abrupt increase in the number of gauche defects within the central region of the chains.

For over 30 years, nuclear magnetic resonance (NMR) has been used to investigate the conformational dynamics of the lipid molecules in membranes. Measurements of the nuclear quadrupole splitting at selectively deuterated methylene groups along the alkyl chains [7,8] have yielded the distribution of gauche defects along the chains in the membrane's fluid phase [8]. Although cold neutron spectroscopy has proved to be a powerful research tool for investigating molecular dynamics on time scales in the range $10^{-13}-10^{-9} \mathrm{~s}$ in many areas of condensed matter research, it has been difficult to apply the technique to biophysical systems such as lipid membranes due to their complexity, small size, and the relatively long time scale of their motions ( $>1 \mathrm{~ns}$ ).

The simpler structure of an alkane monolayer on graphite, a single layer of flexible-chain molecules without headgroups on a well-characterized substrate, motivated us to undertake a study of the molecular conformational dynamics in this system. Here we describe how MD simulations can be combined with highresolution QNS to investigate the intramolecular and rotational diffusive (or "near-zero-frequency") motion in these monolayers associated with the creation and annihilation of gauche defects.

The QNS experiments were performed on the new High Flux Backscattering Spectrometer (HFBS) at the Center for Neutron Research, National Institute of Standards and Technology [9]. The HFBS has a neutron energy resolution of $\sim 1.0 \mu \mathrm{eV}$ and a dynamic range of 


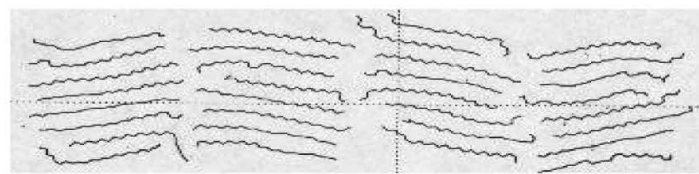

Smectic

$\sim 340 \mathrm{~K}$

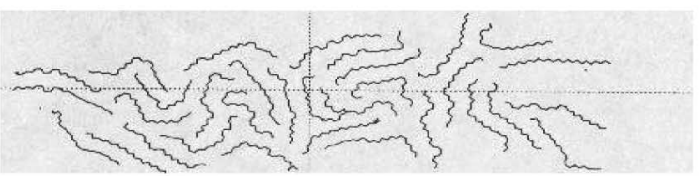

Fluid

FIG. 1. Top view showing configuration of C24 molecules in the MD simulation cell: "smectic" phase (left); fluid phase (right).

0-36 $\mu \mathrm{eV}$. Spectra were collected in a neutron wave vector transfer $(Q)$ range from 0.6 to $1.7 \AA^{-1}$. The large solid angle subtended by the silicon analyzing crystals used to select the final neutron energy makes the HFBS particularly effective for inherently small samples such as adsorbed monolayers.

The sample was prepared by depositing $\mathrm{C} 24$ from its vapor phase onto an exfoliated graphite substrate (Grafoil) in an evacuated cell at a temperature of $\sim 200^{\circ} \mathrm{C}$. It had an annular shape (to reduce multiple scattering) with a height of $30 \mathrm{~mm}$ and inner and outer diameters of $16 \mathrm{~mm}$ and $22 \mathrm{~mm}$, respectively. Preparation details have been described elsewhere [10]. Consistent with earlier studies [6], room temperature neutron diffraction measurements at the University of Missouri Research Reactor on the same sample used for the QNS experiments confirmed the presence of the low-density smectic monolayer C24 phase shown in Fig. 1 (left panel).

In our quasielastic experiments, the $\mathrm{C} 24$ molecules are protonated so that the scattered neutron intensity is completely dominated by the incoherent scattering from the hydrogen which is proportional to $S_{\text {inc }}(Q, \omega)$, the time Fourier transform of the intermediate scattering function $F_{s}(\mathbf{Q}, t)=\frac{1}{N} \sum_{j=1}^{N}\left\langle\exp \left[i \mathbf{Q} \cdot\left(\mathbf{R}_{j}(t)-\mathbf{R}_{j}(0)\right)\right]\right\rangle . F_{s}(\mathbf{Q}, t)$ is, in turn, the space Fourier transform of the selfcorrelation function $G_{s}(\mathbf{r}, t), r=R_{j}(t)-R_{j}(0)$, which gives the probability of finding an atom $j$ at $\mathbf{R}_{j}(t)$ at time $t$ given that the same atom was at $\mathbf{R}_{j}(0)$ at time zero. For diffusive processes that are "slow" on the time scale accessible to the HFBS, $F_{s}(\mathbf{Q}, t)$ decays over many time steps to a constant value contributed by any elastic component in the scattering (zero in its absence). In this case, $S_{\text {inc }}(Q, \omega)$ as observed on the HFBS has a bell shape. On the other hand, for a "fast" process on the HFBS time scale, $F_{s}(\mathbf{Q}, t)$ decays to a constant value in just a few time steps. We can see what effect this has on the observed HFBS spectrum by considering an extreme example in which the time decay is so rapid that it can be described by a $\delta$ function whose Fourier transform yields a flat, frequency-independent contribution to $S_{\text {inc }}(Q, \omega)$. Therefore, it is possible to characterize the observed QNS contributed by a slow process without hindrance from this featureless scattering from any faster motions in the system.

Our strategy in probing diffusive motion related to the creation and annihilation of gauche defects in the C24 molecules is then to use the MD simulations to find a temperature and wave vector range in which all other diffusive motions are fast and, therefore, contribute only a "flat" background to the quasielastic intensity. For this purpose, we have constructed a matrix operator that projects out the center-of-mass motion, the rigid rotational motion, and the intramolecular motion from the displacement of the atoms in a molecule [11]. The intramolecular motion includes vibration, libration, and cooperative motion of atom groups required to create or annihilate a gauche defect.

Figure 2(a) shows the intermediate scattering functions calculated from the MD simulations for the rigid rotational and intramolecular motions at $Q=1.00 \AA^{-1}$ and $260 \mathrm{~K}$. At this temperature, the simulations indicate that there are only a few gauche defects in the molecules and

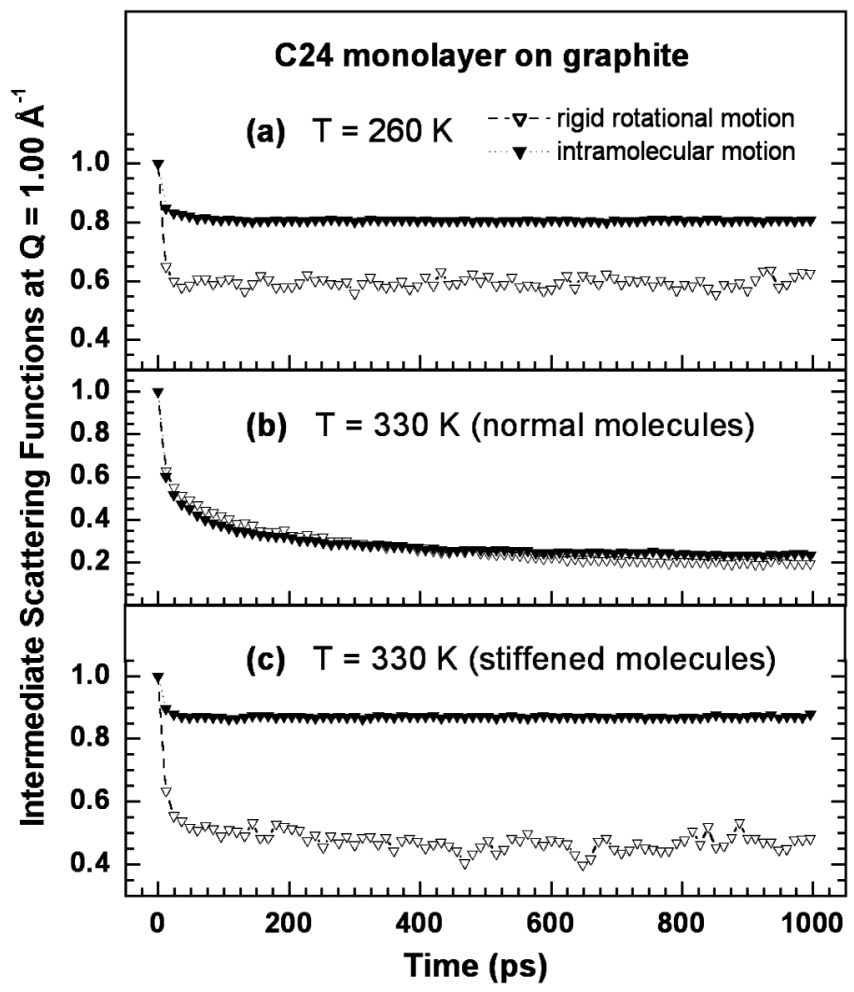

FIG. 2. The intermediate self-scattering function for the rigid rotational and intramolecular motions in the $\mathrm{C} 24$ monolayer calculated from the MD simulation at various temperatures: (a) $260 \mathrm{~K}$; (b) $330 \mathrm{~K}$ (which is $10 \mathrm{~K}$ below the monolayer melting transition); and (c) at $330 \mathrm{~K}$ but with stiffened chains to inhibit formation of gauche defects. 
that these are localized at the chain ends [4]. The open triangles show the contribution to the intermediate scattering function from rotational motion about all three principal axes of the molecule, whereas the closed triangles indicate the intermediate scattering function corresponding to the intramolecular motion after both the rotational motion and the center-of-mass motion have been removed. For both cases, we see that the intermediate scattering function at $260 \mathrm{~K}$ decays rapidly to a constant value in the first few time steps. In this way, we infer that the rigid rotational, vibrational, and librational diffusive motions are too fast to be detected within the dynamic range $(0-36 \mu \mathrm{eV})$ of the HFBS. Furthermore, upon heating, these motions are even faster so that no observable QNS is expected unless a new, slower motion is activated.

In Fig. 2(b), we show the same intermediate scattering functions calculated from the simulation for rigid rotational motion (open triangles) and for the intramolecular motion (solid triangles) as appeared in Fig. 2(a) but at a temperature of $330 \mathrm{~K}$. It is interesting to see that the intermediate scattering functions now decay over many time steps, suggesting that slower rotational and intramolecular motions have been activated. The temperature of $330 \mathrm{~K}$ is $10 \mathrm{~K}$ below the $\mathrm{C} 24$ monolayer melting point where our MD simulations show that, in addition to many molecules having gauche defects at their ends, a significant number of these defects have now appeared in the central region of the alkane chains [4,12]. Thus, it is appealing to interpret these slow intramolecular diffusive motions as associated with the creation and annihilation of gauche defects near the chain centers. Presumably, these motions are slower than for the defects near the chain ends, since they involve the cooperative motion of larger groups of atoms. The slow diffusion indicated by the intermediate scattering function for rigid molecular rotation may also result from a larger number of gauche defects in the molecules' central region. These defects produce a more compact shape of the $\mathrm{C} 24$ molecules as measured, e.g., by their average end-to-end distance $[4,11]$. The slower rotational diffusion can then be explained by the larger moment of inertia about each of the principal molecular axes.

In order to obtain further evidence that the dynamics of gauche defects in the alkane molecules can have an observable effect on the QNS spectra, we have performed MD simulations at $330 \mathrm{~K}$ with stiffened chains to inhibit defect formation. This was accomplished by tripling the trans-to-gauche energy barrier within the C24 molecule and recalculating the intermediate scattering functions. The results shown in Fig. 2(c) indicate that the intermediate scattering functions again decay in the first few time steps so that the slow diffusive motions have disappeared as anticipated.

For comparison with these simulations, we conducted quasielastic measurements beginning at low tempera- ture where we anticipated that the molecular diffusive motion would be simplest to describe. Upon heating the sample to a temperature of $\sim 215 \mathrm{~K}$, just above the monolayer crystalline-to-smectic phase transition [13], we observe QNS with sufficient intensity to determine the $Q$ dependence of its energy width [14]. A typical spectrum was shown in an earlier paper [15]. At each $Q$, the quasielastic intensity is fit to the sum of a Lorentzian and a $\delta$ function convoluted with the instrumental resolution function. The best-fit values of the Lorentzian intensity and its half width at half maximum (HWHM) were found to be insensitive to the initial values assumed. We see in Fig. 3 that the Lorentzian HWHM is essentially dispersionless at temperatures of 215 and $230 \mathrm{~K}$.

Snapshots of the molecular motions made from our simulations show that the diffusive motion at low temperature is dominated by a uniaxial rotation of the molecules about their long axis aligned parallel to the graphite surface. Such a model is also consistent with both the $Q$ dependence of the constant level of the intermediate scattering function at long times calculated from the MD simulations as well as the observed dispersionless HWHM of the Lorentzian component of the QNS at 215 and $230 \mathrm{~K}$. Fitting the observed quasielastic spectra at $230 \mathrm{~K}$ in Fig. 3 to this model of uniaxial rotation, we find a time constant of $400 \pm 100 \mathrm{ps}$ for this motion [15]. The large uncertainty reflects a corresponding uncertainty in the average distance of the methylene groups from the long molecular axis due to the presence of gauche defects.

At $260 \mathrm{~K}$, the energy width of the QNS increases with $Q$ as shown in Fig. 3. Our simulations suggest that this $Q$ dependence results primarily from nonuniaxial rotational motion and, to a lesser degree, translational diffusion. However, the simulation predicts a temperature range between 260 and $300 \mathrm{~K}$ in which rotational and intramolecular diffusive motion is sufficiently rapid to be

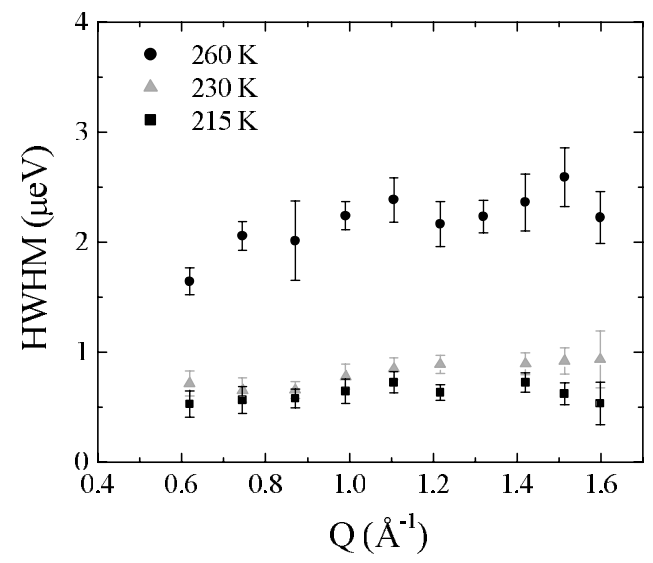

FIG. 3. Results of fitting the quasielastic spectra at low temperatures: the energy width of the Lorentzian component (half width at half maximum) is plotted versus wave vector transfer $Q$. 


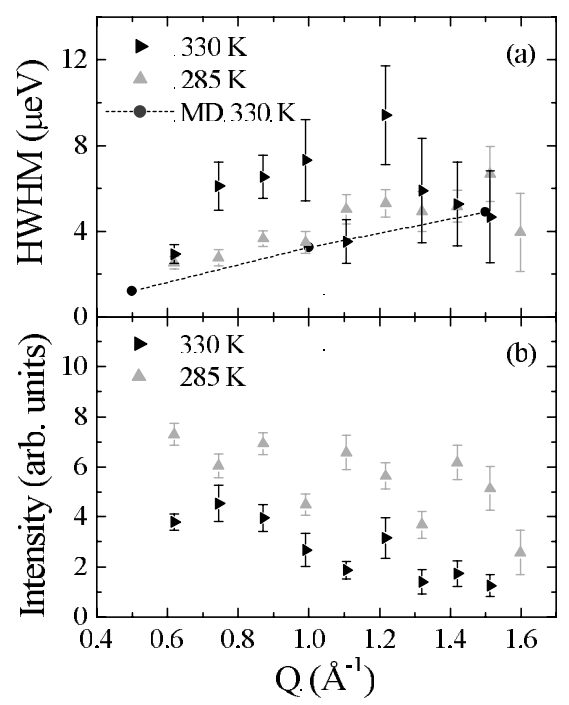

FIG. 4. Results of fitting the quasielastic spectra at higher temperatures: (a) the energy width of the Lorentzian component vs wave vector transfer $Q$; (b) the intensity of the Lorentzian component vs $Q$. In (a), the dashed line indicates values calculated from the MD simulations at $330 \mathrm{~K}$.

outside the energy window of the spectrometer [see Fig. 2(a)]. We believe that this is not observed experimentally because the unified-atom representation of the molecule in the simulation [4] underestimates the moment of inertia about the long molecular axis by about a factor of 2 , resulting in faster rotational diffusion. Such an error in the moment of inertia does not affect the simulation of slower motions associated with gauche defect creation in the central region of the $\mathrm{C} 24$ chains.

Our measurements support the prediction of the MD simulations that QNS should be observable within the energy window of the HFBS at $330 \mathrm{~K}$ due to gauche defect production. In Fig. 4, we have analyzed the $Q$ dependence of the HFBS spectra at temperatures of 285 and $330 \mathrm{~K}$ in the same way as for lower temperatures (see Fig. 3): (a) the Lorentzian HWHM characterizing the energy width of the observed QNS; and (b) the relative intensity of the Lorentzian component in the spectra. Although the intensity becomes very weak at $330 \mathrm{~K}$ resulting in larger error bars than at lower temperature, it is clear that there is still QNS within the dynamic range of the HFBS. Comparing with Fig. 3, we see that at each $Q$ the energy width of the QNS continues to increase with temperature above $260 \mathrm{~K}$. There is also a large increase in the slope of the HWHM vs $Q$ between 260 and $285 \mathrm{~K}$. For comparison, we have also plotted in Fig. 4 (dashed line) the HWHM of a Lorentzian fitted to $S_{\text {inc }}(Q, \omega)$ calculated from the MD simulation at $330 \mathrm{~K}$ after removing the center-of-mass motion from the $\mathrm{C} 24$ molecules. These calculated HWHM's characterize the contribution to the QNS from both rotational and intramolecular diffusive motions. We conclude that both their magnitude and $Q$ dependence are in qualitative agreement with experiment.

In summary, our experiments demonstrate that neutron spectrometers such as the HFBS, combining both high energy resolution with large throughput, can be used to investigate molecular conformational dynamics on a nanosecond time scale in monolayer films. In addition to the dynamical time scales of interest, such measurements also probe length scales relevant to the motion through the observed $Q$ dependence of the QNS, a characteristic of the motion that is inaccessible by NMR techniques. This $Q$ dependence may not be describable by simple models of translational and rotational diffusion but is calculable from MD simulations as we have illustrated.

This work was supported by the NSF under Grants No. DMR-9802476 and No. DMR-0109057, by the Chilean government under FONDECYT Grant No. 1010548, and by the U.S. Department of Energy through Grant No. DE-FG02-01ER45912. The neutron scattering facilities in this work are supported in part by the National Science Foundation under Agreement No. DMR-0086210.

*Present address: Infineon Technologies, Memory Products, Balanstrasse 73, D-81541 Munich, Germany.

[1] T. K. Xia et al., Phys. Rev. Lett. 69, 1967 (1992).

[2] T. K. Xia and Uzi Landman, Science 261, 1310 (1993).

[3] Uzi Landman et al., Jpn. J. Appl. Phys. 32, 1444 (1993).

[4] F. Y. Hansen et al., Phys. Rev. Lett. 83, 2362 (1999).

[5] D. M. Small, The Physical Chemistry of Lipids: From Alkanes to Phospholipids (Plenum, New York, 1986), p. 523.

[6] K.W. Herwig, B. Matthies, and H. Taub, Phys. Rev. Lett. 75, 3154 (1995).

[7] For a recent review see, e.g., R.W. Pastor, R. M. Venable, and S. E. Feller, Acc. Chem. Res. 35, 438 (2002).

[8] J.-P. Douliez, A. Léonard, and E. J. Dufourc, Biophys. J. 68, 1727 (1995).

[9] A. Meyer et al., Rev. Sci. Instrum. 74, 2759 (2003).

[10] K.W. Herwig, B. Matthies, and H. Taub, in Neutron Scattering in Materials Science, edited by D. A. Neumann, T. P. Russell, and B. J. Wuensch, Materials Research Society Symposium Proceedings Vol. 376 (Materials Research Society, Pittsburgh, PA, 1994), p. 757.

[11] F. Y. Hansen (unpublished).

[12] Figure 4 of Ref. [4] contains distributions of gauche bonds along a molecular chain in a C32 monolayer. Similar distributions have been calculated from MD simulations of C24 monolayers [F.Y. Hansen, K.W. Herwig, and H. Taub (unpublished)].

[13] B. Matthies et al. (unpublished).

[14] For example, at $260 \mathrm{~K}$ the ratio of the integrated quasielastic intensity to elastic intensity is $\sim 0.7$ at $Q=1.0 \AA^{-1}$.

[15] K.W. Herwig et al., J. Phys. IV (France) 10, 157 (2000). 